ESJ Social Sciences

\title{
Clima-Organizacional Estudiantil en Contexto de Diversidad Cultural, Aplicando un Modelo de Correlación en el Norte de Sinaloa
}

\author{
PDr. Oscar Alejandro Viramontes-Olivas \\ Profesor-Investigador, Facultad de Contaduría y Administración, \\ Universidad Autónoma de Chihuahua, México \\ PDr. Ernesto Guerra García \\ Profesor-Investigador, Universidad Autónoma Indígena de México \\ Dr. René Arroyo Ávila \\ Profesor-Investigador, Facultad de Contaduría y Administración, \\ Universidad Autónoma de Chihuahua, México \\ Dra. María del Rosario de Fátima Alvidrez Díaz \\ Profesora-Investigadora, Facultad de Contaduría y Administración, \\ Universidad Autónoma de Chihuahua, México

\section{M.A.R.H. Cesar Báez Terrazas} \\ Profesor-Investigador, Facultad de Contaduría y Administración, \\ Universidad Autónoma de Chihuahua, México
}

Doi:10.19044/esj.2021.v17n23p117

Submitted: 04 May 2021

Accepted: 01 July 2021

Published: 31 July 2021
Copyright 2021 Author(s)

Under Creative Commons BY-NC-ND

4.0 OPEN ACCESS

Cite As:

Viramontes-Olivas O. A., García E.G., Ávila R.A., Alvidrez Díaz M.R.F. \& Terrazas C.B. (2021). Clima-Organizacional Estudiantil en Contexto de Diversidad Cultural, Aplicando un Modelo de Correlación en el Norte de Sinaloa. European Scientific Journal, ESJ, 17(23), 117. https://doi.org/10.19044/esj.2021.v17n23p117

\section{Resumen}

El Clima organizacional (Co) es importante para estimular, impulsar y mejorar la productividad del Recurso humano (Rh) que genera, crea, promueve, gestiona y mantiene constante innovación para el desarrollo grupal e individual. Se analizaron condiciones de Co en estudiantes de tres unidades académicas de una Universidad intercultural en Sinaloa para detectar, fortalezas, debilidades, oportunidades y amenazas. Se aplicaron 1,176 encuestas (escala Likert-siete niveles), con 70 preguntas, siendo validada por alfa de Cronbach (0.86). El muestreo fue por Método Aleatorio Estratificado, Afijación Proporcional con nivel de confianza (97\%) y error $(2.8 \%)$ para 
$\mathrm{N}=5,375$. Se estratificó por unidad y carrera, en el caso de Los Mochis, se tuvo una muestra de $(n=703)$; Mochicahui, $(n=361)$ y Choix, $(n=112)$. Las variables medidas fueron: Servicio a estudiantes (Se), Respeto a la Diversidad Cultural (Rdc), Cohesión (Cn), Gestión (Gn), Área Física (Af) y Equidad (Eq). Se aplicó el Coeficiente de Correlación (Pearson) con regresión bivariada y multivariada. Se fue estadísticamente significativa con $\mathrm{Co}(\mathrm{r}=0.787 \mathrm{P}<0.01)$; Co con $\mathrm{Rdc}(\mathrm{r}=0.761 \mathrm{P}<0.01)$; Co con $\mathrm{Cn}(\mathrm{r}=0.832 \mathrm{P}<0.01)$; Co con $\mathrm{Gn}$ $(\mathrm{r}=0.834 \mathrm{P}<0.01)$; Co con Af $(\mathrm{r}=0.796 \mathrm{P}<0.01)$ y Co con $\mathrm{Eq}(\mathrm{r}=0.820 \mathrm{P}<0.01)$. La R2 fue de $91.8 \%$. De acuerdo al predictor de las seis variables analizadas las más significativas en orden de importancia fueron: Af, Se, Gn, Eq, Rdc y $\mathrm{Cn}$, para la toma de decisiones. Las fortalezas son que la Universidad es incluyente; sus debilidades son: existe una organización y gobierno "vertical", fragmentando la unidad; amenazas, desunión y falta de comunicación; oportunidades, sus programas están certificados y con calidad educativa.

Palabras claves: Convivencia, derechos humanos, desarrollo, diversidad e integración, regresión líneal

\title{
Student Organizational-Climate in the Context of Cultural Diversity, Applying a Correlation Model in the North of Sinaloa
}

\author{
PDr. Oscar Alejandro Viramontes-Olivas \\ Profesor-Investigador, Facultad de Contaduría y Administración, \\ Universidad Autónoma de Chihuahua, México \\ PDr. Ernesto Guerra García \\ Profesor-Investigador, Universidad Autónoma Indígena de México

\section{Dr. René Arroyo Ávila} \\ Profesor-Investigador, Facultad de Contaduría y Administración, \\ Universidad Autónoma de Chihuahua, México \\ Dra. María del Rosario de Fátima Alvidrez Díaz \\ Profesora-Investigadora, Facultad de Contaduría y Administración, \\ Universidad Autónoma de Chihuahua, México \\ M.A.R.H. Cesar Báez Terrazas \\ Profesor-Investigador, Facultad de Contaduría y Administración, \\ Universidad Autónoma de Chihuahua, México
}

Abstract

Organizational climate $(\mathrm{Co})$ is important to stimulate, boost and improve the productivity of the Human Resource $(\mathrm{Rh})$ that generates, 
promotes, manages, creates and maintains constant innovation for group and individual development. Objective. Co conditions were analyzed in students of three academic units of an intercultural University in Sinaloa, to detect, strengths, weaknesses, opportunities and threats. Method. 1,176 surveys were applied (Likert scale-seven levels), with 70 questions, being validated by Cronbach's alpha (0.86). Sampling was by Stratified Random Method, Proportional Locking, with confidence level $(97 \%)$ and error $(2.8 \%)$ for $\mathrm{N}=$ 5,375 . It was stratified by unit and career, in the case of Los Mochis, there was a sample of $(n=703)$; Mochicahui, $(n=361)$ and Choix, $(n=112)$. The variables measured were: Student service (Se), Respect for Cultural Diversity (Rdc), Cohesion (Cn), Management (Gn), Physical Area (Af) and Equity (Eq) Results The Correlation Coefficient (Pearson) was applied, with bivariate and multivariate regression. It was statistically significant with $\mathrm{Co}(\mathrm{r}=0.787 \mathrm{P}$ $<0.01)$; Co with Rdc $(\mathrm{r}=0.761 \mathrm{P}<0.01)$; Co with $\mathrm{Cn}(\mathrm{r}=0.832 \mathrm{P}<0.01)$; Co with $\mathrm{Gn}(\mathrm{r}=0.834 \mathrm{P}<0.01)$; Co with Af $(\mathrm{r}=0.796 \mathrm{P}<0.01)$ and Co with Eq $(\mathrm{r}=0.820 \mathrm{P}<0.01)$. $\mathrm{R} 2$ was $91.8 \%$. According to the predictor of the six variables analyzed the most significant in order of importance were: Af, Se, $\mathrm{Gn}, \mathrm{Eq}, \mathrm{Rdc}$ and $\mathrm{Cn}$, for decision-making. The strengths are that the University is inclusive; its weaknesses are: there is a "vertical" organization and government, fragmenting the unit; threats, disunity and lack of communication; opportunities, its programs are certified and with educational quality.

Keywords: Coexistence, human rights, development, diversity and integration, linear regression

\section{Introduction}

Según Castillo y Guido (2015), la diversidad cultural es la evolución y transformación de las sociedades han influido en la presencia de conflictos armados en la defensa de los derechos culturales personales, sobre todo, para buscar el respeto a creencias, formas de pensar y hacer, parten de su existencia en una diversidad cultural marcada en América Latina. Núñez y Córdoba (2020) indican que desde finales del siglo XX y principios del XXI, estas luchas buscaron la reivindicación de los pueblos por décadas o siglos, debido a que estaban olvidados y marginados de los escenarios nacionales e internacionales, simplemente por pertenecer a determinado grupo social y debido a la globalización de la economía que avanza en la sociedad actual que ha contribuido a la pérdida de la diversidad cultural ancestral y la falta de oportunidades para cientos de personas (Figueroa-Céspedes y Yáñez-Urbina, 2020). González (2017), el Clima organizacional (Co) en un entorno intercultural, enfocando el significado a un conjunto de atributos, propiedades y cualidades evidentes en cada persona o grupo, practicados en cualquier 
ambiente organizado e influenciado por componentes de la comunidad, llegando a repercutir en la conducta, valores, identidad y en la cultura en general. En el caso de la existencia de un ambiente plural con diversidad cultural como se puede apreciar en la sociedad actual, exige nuevas y mejores estrategias políticas, económicas, sociales, culturales y educativas para buscar el progreso. El reconocimiento y respeto a las universitarias a partir de la diversidad cultural, Cabrera y Gallardo (2013) mencionan que el compromiso más urgente y complejo que tienen que enfrentar las Instituciones de Nivel Superior (INS) en México, son los cambios tendientes al respeto y reconocimiento de la diversidad dentro del Co para mitigar conductas discriminatorias de género, posición económica, etnia, origen, religión, discapacidad física y el papel cultural en la sociedad, promoviendo mayor desarrollo entre estudiantes y lograr el éxito del proceso enseñanzaaprendizaje.

González, Ortíz y Gamarra (2020) señalan que definir el Co de forma única no es correcto, ya que incluye diversos conceptos y expresiones que inciden sobre personas o grupos en la promoción de la mejora continua, tendientes a lograr calidad en los procesos de enseñanza para alcanzar el éxito. El Co está determinado por conductas, actitudes, aptitudes, expectativas y realidades sociológicas, económicas y culturales de la organización. Se puede decir que representa la diversidad de variables situacionales que buscan promover el orden, disciplina y naturaleza del ambiente donde se quiere implantar, la cual, se puede ir transformando con el tiempo, afectando o beneficiando directa e indirectamente a los involucrados, más aún, cuando se busca la equidad ya que la marginación y discriminación, influyen en el dinamismo laboral-organizacional y académico en las universidades. Por ello, se busca la continuidad programática en ambientes con características de diversidad estudiantil sin importar el origen racial o de grupo (Bolaños et al., 2020).

Alemán y Torres (2020) mencionan que la buena gestión del Recurso humano $(R h)$ en universidades, mejora la calidad del $C o$ en el entorno donde interaccionan las personas. El impacto de la gestión dentro de un escenario intercultural, se ve afectado por el ambiente que domina y otros factores como la cultura. En diversas instituciones existen situaciones desmotivantes que influyen en un bajo compromiso de sus integrantes a las políticas de mejora, llegándose a implementar prácticas de alto desempeño que generen sinergia en la base y así, aumentar dicho compromiso que motive y mejoren los resultados académicos a corto, mediano y largo plazo. Sánchez, Hernández y Jiménez (2016) concluyen que un $R h$ bien administrado y capacitado, se convertirá en un activo poderoso, incluso una ventaja competitiva por lo que buen número de universidades están expuestas a cambios dinámicos y dependen de sí mismas para estar preparadas y sobrevivir ante amenazas del 
exterior, formulando la gestión radicalmente para ser más innovadoras, adaptadas y flexibles en el logro de resultados.

Con base en lo anterior, el objetivo de la presente investigación fue analizar las condiciones de Clima organizacional $(\mathrm{Co})$ en estudiantes de una Universidad dentro de un contexto de diversidad cultural con el fin de detectar fortalezas, debilidades, oportunidades y amenazas en sus tres unidades académicas (Mochis, Mochicahui y Choix) en el norte de Sinaloa.

\section{Hipótesis}

Ho Hipótesis nula. Fomentar en estudiantes universitarios ambientes de mejoramiento en el Clima organizacional dentro de un contexto de diversidad cultural, no impulsará el mejoramiento en la calidad de planes educativos, ni propiciará la formación integral de profesionales competentes, críticos y comprometidos con el desarrollo sustentable de sus comunidades.

$H_{a}$ Hipótesis alterna. Fomentar en estudiantes universitarios ambientes de mejoramiento en el Clima organizacional dentro de un contexto de diversidad cultural, impulsará el mejoramiento en la calidad de planes educativos y propiciará la formación integral de profesionales competentes, críticos y comprometidos con el desarrollo sustentable de sus comunidades.

\section{Materiales y métodos}

La Universidad donde se desarrolló la investigación es una Institución del norte del estado de Sinaloa, México con tres unidades académicas (Los Mochis, Mochicahui y Choix), cuya misión es propiciar la formación integral de profesionales competentes, críticos y comprometidos con el desarrollo sustentable, orientadas a la investigación en la solución de problemas sociales y la difusión y extensión de servicios en un contexto de diversidad cultural, llevándose a cabo esta investigación de noviembre de 2018 a noviembre de 2020.

Instrumento de encuesta. Se aplicó un instrumento de encuesta a partir de la escala de medición de Likert con siete niveles (Boone and Boone, 2012) mostrándose en la (Tabla 1).

Validez y confiabilidad (experto y campo). Se llevaron entrevistas a un grupo de 13 personas de cada unidad académica (Los Mochis, Mochicahui y Choix), los que no fueron incluidos en la muestra de estudio (prueba piloto) garantizando condiciones para el trabajo real y calculada con el alfa de Cronbach para validarla (Corral, 2009). 
Tabla 1. Especificaciones de la escala de medición Likert con diferentes niveles (1 al 7), integrados en instrumentos de encuesta a estudiantes en un contexto de diversidad cultural universitario en el norte de Sinaloa.

\begin{tabular}{lc}
\hline \multicolumn{1}{c}{ Escala de medición } & Nivel/Valor \\
\hline Muy insatisfecha/muy en desacuerdo. & 1 \\
Insatisfecho/en desacuerdo. & 2 \\
Moderadamente insatisfecha/moderadamente en desacuerdo. & 3 \\
Ni satisfecha ni de acuerdo. & 4 \\
Moderadamente satisfecho/moderadamente de acuerdo. & 5 \\
Satisfecho/de acuerdo. & 6 \\
Muy satisfecho/muy de acuerdo. & 7 \\
\hline
\end{tabular}

*Fuente: Elaboración propia (2021).

Validez y confiabilidad (experto y campo). Anderson et al. (2019) sugiere la siguiente fórmula para establecer el grado de validez y confiabilidad de la prueba de alfa Cronbach.

$$
\propto=\frac{K}{K-1}\left(1-\frac{\sum V i}{V t}\right)
$$

Ecuación alfa de Cronbach

\section{Donde}

Alfa= alfa de Cronbach.

$K=$ Número de ítems.

Vi= Varianza de cada ítems.

$V t=$ Varianza del total .

El valor de alfa de Cronbach se determinó empleando el programa SPSS y de acuerdo con la escala de Likert 7 niveles, el resultado del índice fue de 0.86, por lo que fue consistente para esta escala, mostrándose en el (Tabla 2).

Tabla 2. Valor de alfa de Cronbach calculado para validar la encuesta.

\begin{tabular}{cccc}
\hline Escala Likert & Varianza de Ítems & SPSS & Alfa estandarizado \\
\hline Nivel 7 & 0.88 & 0.88 & 0.86 \\
\hline \multicolumn{4}{c}{ *Fuente: Elaboración propia (2021). }
\end{tabular}

Descripción de la población en estudio. Se consideró la distribución de estudiantes por programa académico (licenciatura y posgrado), distribución por unidad académica y carrera (Tabla 3 ).

Tabla 3. Distribución de estudiantes universitarios en tres unidades académicas de una Universidad en un contexto de diversidad cultural.

\begin{tabular}{lc}
\hline \multicolumn{1}{c}{ Programa Académico } & Los Mochis \\
\hline Licenciado en Educación Preescolar & 261 \\
Licenciado en Psicología Social Comunitaria & 549 \\
Licenciado en Sociología Rural & 59 \\
Licenciado en Derecho (escolarizado) & 475 \\
Licenciado en Contaduría & 566
\end{tabular}




\begin{tabular}{|c|c|}
\hline Total & 3,214 \\
\hline $\begin{array}{l}\text { Programa Académico } \\
\end{array}$ & Mochicahui \\
\hline Licenciado en Psicología Social Comunitaria & 241 \\
\hline Licenciado en Turismo Empresarial & 125 \\
\hline Licenciado en Sociología Rural & 248 \\
\hline Licenciado en Derecho (escolarizado) & 240 \\
\hline Licenciado en Contaduría & 186 \\
\hline Ingeniero en Sistemas Computacionales & 231 \\
\hline Ingeniero en Sistemas de Calidad & 242 \\
\hline Ingeniero Forestal & 135 \\
\hline Total & 1648 \\
\hline $\begin{array}{l}\text { Programa Académico } \\
\end{array}$ & Choix \\
\hline Ingeniero en Sistemas Computacionales & 267 \\
\hline Ingeniero Forestal Comunitario & 159 \\
\hline Licenciado en Turismo Alternativo & 87 \\
\hline $\begin{array}{ll} & \text { Total } \\
\end{array}$ & 513 \\
\hline Posgrado & 250 \\
\hline
\end{tabular}

Variables contempladas. Para la consulta estudiantil sobre condiciones de Clima organizacional $(\mathrm{Co})$ en un contexto de diversidad cultural, se contemplaron las siguientes variables: Servicio a estudiantes $(\mathrm{Se})$, Respeto a la diversidad cultural $(R d c)$, Cohesión $(C n)$, Gestión $(G n)$, Área física $(A f)$ y Equidad estudiantil $(E q)$.

Obtención de la muestra. Con base en las características y distribución de la población y para la obtención de la muestra se utilizó el Muestreo Aleatorio Estratificado por Afijación Proporcional:

$$
n=\frac{\sum{ }^{n_{h=}}\left(W_{h} P_{h\left(1-P_{h}\right)}\right.}{D+\frac{1}{N} \sum W_{h} P_{h}(1-P h)}
$$

(Anderson et al., 2019) *El tamaño de "n" para un margen de error "e" con un nivel de confianza.

Nivel de confianza y error máximo en la muestra estudiantil. La población estudiantil en las tres unidades fue $\mathrm{N}=5,375$, distribuidos en 19 programas académicos. Para el cálculo de la misma se consideró un nivel de confianza de $97 \%$ con margen de error máximo admitido de $2.8 \%$, obteniéndose ( $\mathrm{n}=1,176$ alumnos), bajo el supuesto de que $\mathrm{p}=\mathrm{q}=50 \%$ con un número de estratos de tres por considerar las unidades académicas (Tabla 4): 
Tabla 4. Tamaño de muestra aleatoria estratificada por afijación proporcional global por estrato (unidades académicas), con un nivel de confianza (97\%) y error máximo admitido de $2.8 \%$ en estudiantes.

\begin{tabular}{ccccc}
\hline Estrato & Unidad & Número de Alumnos por Unidad & Proporción & $\begin{array}{c}\text { Muestra del } \\
\text { Estrato }\end{array}$ \\
\hline 1 & Los Mochis & 3,214 & $59.8 \%$ & 703 \\
2 & Mochicahui & 1,648 & $30.7 \%$ & 361 \\
3 & Choix & 513 & $9.5 \%$ & 112 \\
\hline & Total & $\mathbf{5 , 3 7 5}$ & $\mathbf{1 0 0 \%}$ & $\mathbf{1 , 1 7 6}$ \\
\hline
\end{tabular}

*Fuente: Elaboración propia (2021).

\section{Nivel de confianza y error máximo en la muestra}

Análisis de datos. Para el análisis de los datos bivariados y su relación entre variables, se instrumentó el Coeficiente de Correlación de Pearson $(r)$ para muestras y variables cuantitativas (escala mínima de intervalo), método paramétrico donde se consideró la media y varianza, por tanto, se requirió de criterios de normalidad entre distintas variables relacionadas linealmente ya que se pretendió saber si existen variables fuertemente correlacionadas.

Y la fórmula:

$$
0 \leq r x y \leq 1
$$

$$
r_{x y}=\sum_{i=1}^{n}=\frac{1\left(x_{1}-\underline{x}\right)\left(y_{1}-\underline{y}\right)}{\sqrt{\sum_{i=1}^{n}\left(x_{1}-\underline{x}\right) \dot{2}} \sum_{i=1}^{n}\left(y_{1}-\underline{y}\right)^{2}}
$$

(Anderson et al., 2014)

Se llevó un análisis de regresión simple y múltiple para modelar la relación existente entre la variable explicativa $(X)$ y la variable respuesta $(Y)$ donde:

$$
\begin{gathered}
Y i^{m}=a+\beta i X_{i} \\
Y i^{m}=a+\beta i X_{i}+\cdots \beta i X_{n}
\end{gathered}
$$

Programas para análisis de datos. Para el manejo, acopio, estructuración de datos y análisis estadístico de los datos, se utilizaron dos softwares: Excel de Microsoft Windows, versión (2016) y el programa IBMSPSS Statistics versión 22 (2013). Finalmente para la escritura del trabajo se utilizó el procesador de palabras Word de Microsoft Windows, versión (2013). 


\section{Resultados}

Edad. La distribución de alumnos por edad (Figura 1) donde predominaron jóvenes entre $15-20$ años (43.6\%) y 21 a 35 años (44.6\%), respectivamente. La muestra de adultos (36 a 60 años) fue de $11.8 \%$, según clasificación de Castillo (2015).

Figura 1. Distribución de estudiantes por Edad en tres unidades académicas (Los Mochis, Mochicahui y Choix), Sinaloa.

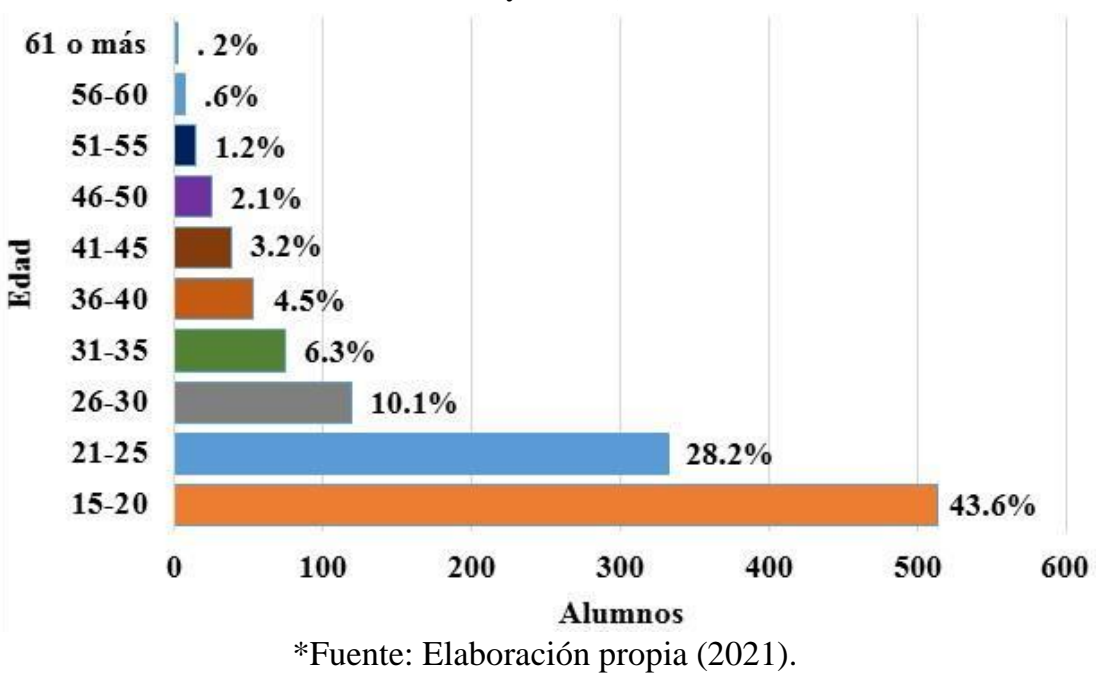

Género. Los datos se ilustran en la (Figura 2), donde las mujeres son mayoría (56.8\%), mientras que los varones representaron $43.2 \%$.

Nivel de estudios. En la (Figura 3) se exponen los resultados de la distribución de estudiantes por nivel académico. El 93.2\% son de licenciatura en sus diversos programas educativos y $6.8 \%$ de posgrado.

Figura 2. Distribución por Género en estudiantes en tres unidades académicas (Los Mochis, Mochicahui y Choix), Sinaloa.

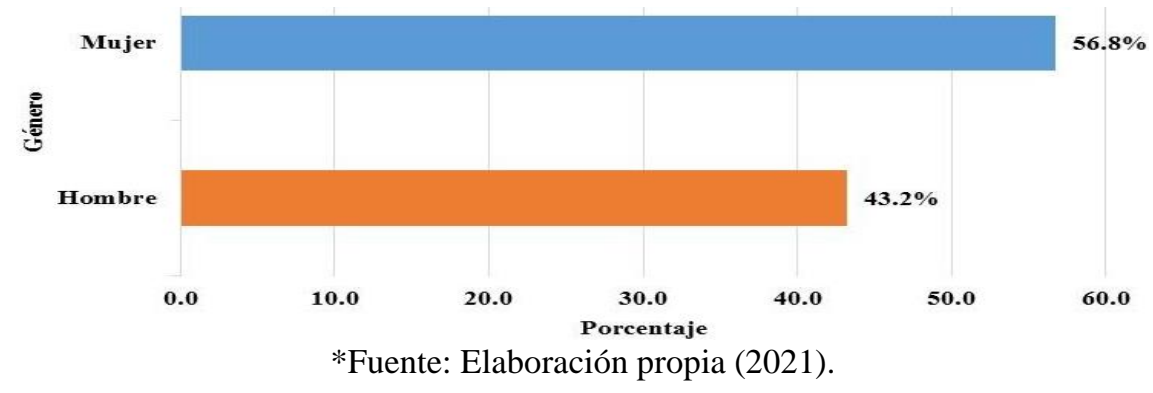


Figura 3. Distribución por Nivel Educativo en estudiantes en las tres unidades académicas (Los Mochis, Mochicahui y Choix); Sinaloa.

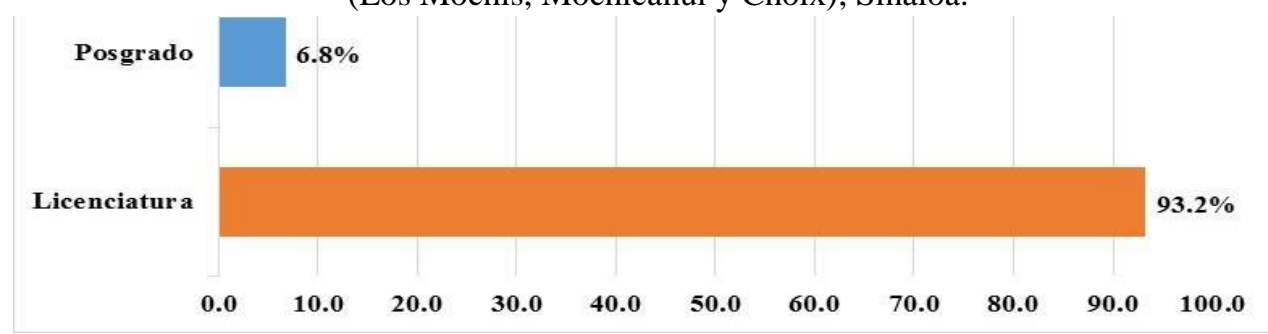

*Fuente: Elaboración propia (2021).

Lengua y pertenencia a grupo originario. De las tres unidades académicas $87.4 \%$ dijeron no hablar alguna lengua originaria sólo el español, aunque algunos argumentaron pertenecer algún grupo étnico pero no saben hablar la lengua materna; $85 \%$, no pertenecen a ninguna y son llamados "mestizos o yories"; $15 \%$ son de alguna etnia, siendo mayoría los mayos-yoremes con $8.3 \%$ (Figura 4).

Figura 4. Distribución de estudiantes por Lengua y Grupo Étnico en tres unidades académicas (Los Mochis, Mochicahui y Choix).

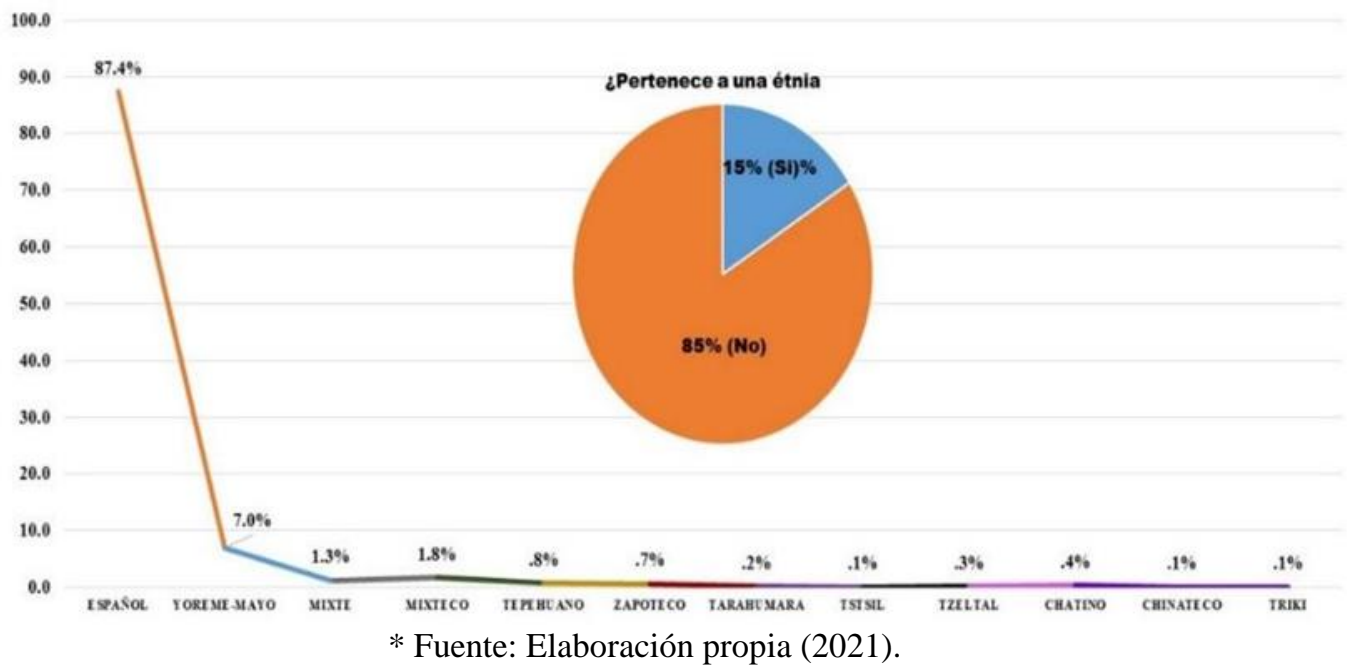

Estado civil. En la actualidad los estudiantes enfrentan distintas realidades que los motivan a esforzarse en actividades más allá de las aulas. Algunos han contraído matrimonio, viven en unión libre y en otras circunstancias, aparte del espacio destinado al trabajo para solventar necesidades básicas familiares o individuales donde 56.6\%, trabajan para resolver diversos compromisos; $22.1 \%$ están casados, lo que influye en el rendimiento académico y su probable deserción escolar. La mayoría son solteros (74.1\%), algunos trabajan y otros no. Aquellos con alguna actividad extra clases $(33.3 \%)$, laboran en la iniciativa privada; $12.4 \%$, (sector público); 
$5.3 \%$ (el campo); $7.1 \%$ (otras actividades) y $41.2 \%$ dedicados solo a la escuela (Tabla 5).

Tabla 5. Actividades extra clase en estudiantes de tres unidades académicas (Los Mochis, Mochicahui y Choix), Sinaloa.

\begin{tabular}{cc}
\hline Actividad Extra Clase & Porcentaje \\
\hline Negocio propio. & 3.8 \\
Iniciativa privada. & 33.3 \\
Sector público & 12.4 \\
Hogar & 3.0 \\
Campo & 5.3 \\
No trabaja & 41.2 \\
Otras actividades & 1.0 \\
\hline Total & $\mathbf{1 0 0}$ \\
\hline
\end{tabular}

*Fuente: Elaboración propia (2021).

\section{Resultados de Variables Estructurales.}

Servicio a estudiantes (Se). En la (Figura 5) 50.6\% está de satisfecho a muy satisfecho al encontrar interacción entre alumnos, administrativos y académicos; moderadamente satisfechos (31.7\%); alumnos que no les interesa si se les proporcionan o no un buen servicio (11.3\%) y $6.4 \%$ están desde muy a moderadamente insatisfechos, quienes expresan que nunca se les ha tomado en cuenta ya que la Universidad no fomenta actividades grupales que mejoren la interacción entre jóvenes, argumentando que existen pocas actividades de convivencia.

Correlación entre (Se) y Clima organizacional (Co). Los resultados entre $\mathrm{Se}$ y $\mathrm{Co}$ aplicando un modelo de Correlación Lineal de Pearson fueron estadísticamente significativo $(r=0.787 \quad P<0.01)$, resultado del grado de asociación entre las variables (Figura 6). De forma particular, Se comparada con Cohesión $(r=0.684 P<0.01)$, Gestión $(r=0.653 P<0.01)$, Área física $(r=0.558 \quad P<0.01) \quad$ y Equidad estudiantil $(r=0.625 \quad P<0.01)$ fueron moderadamente significativas, reflejo que si la Universidad aumenta las expectativas y satisfactores para los jóvenes con un servicio de calidad, el $C o$ tendería a ser mejor y a fortalecerse. 
Figura 5. Opinión sobre Servicio a estudiantes ( $\mathrm{Se}$ ) que reciben los estudiantes en la Universidad.

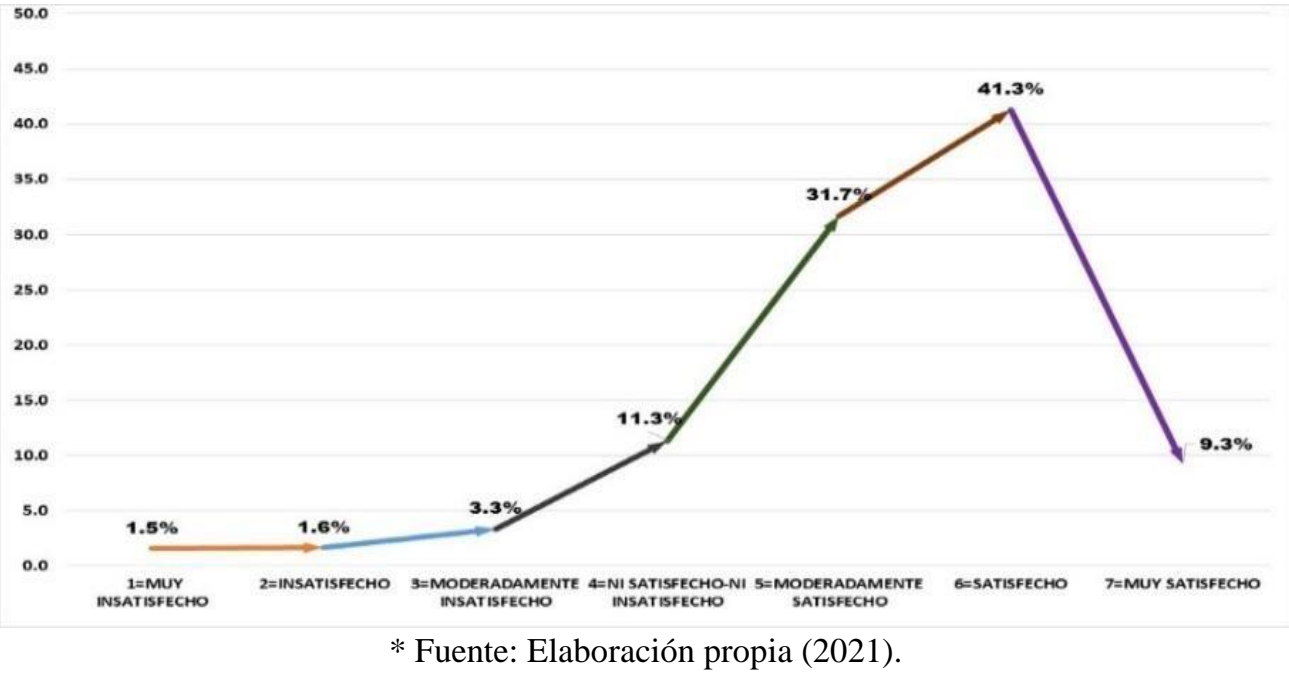

Figura 6. Correlación estadísticamente significativa $(r=0.787 P<0.01)$ entre variables $S e$ con $C o$, en estudiantes de una Universidad con enfoque de diversidad cultural.

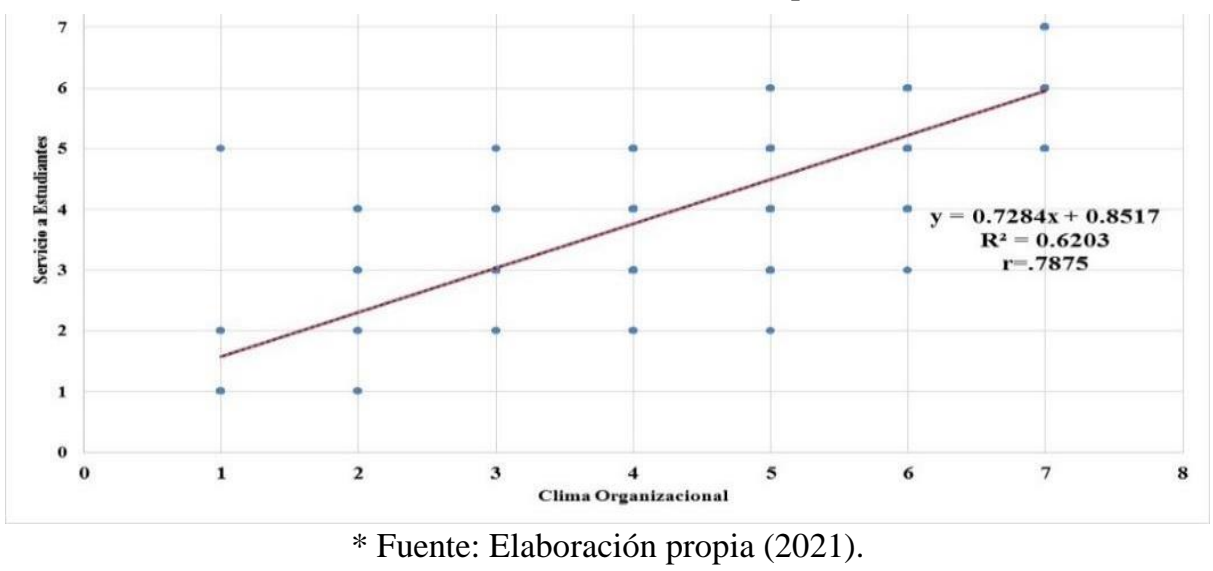

Respeto a la diversidad cultural (Rdc). El 63.6\% está de muy a satisfecho de cómo se fomentan los valores y el respeto a la diversidad cultural; 27.4\%, moderadamente satisfecho ya que algunos no se comprometen o involucran en cursos, talleres, congresos que tengan que ver con el $\boldsymbol{R} \boldsymbol{d} \boldsymbol{c}$ y la falta de compromiso de directivos y maestros por atender estos temas; $6.0 \%$, están moderadamente insatisfechos y $2.9 \%$ insatisfechos (Figura 7).

Correlación entre $R d c$ y Co. Fue estadísticamente significativa $(r=0.761 \quad P<0.01)$, Figura 8. El Rdc vs Servicio a estudiantes ( $r=0.658$ $P<0.01)$, Cohesión ( $r=0.627 P<0.01)$, Gestión $(r=0.564 P<0.01)$, Área física $(r=0.563 \quad P<0.01) \quad$ y Equidad estudiantil $(r=0.642 \quad P<0.01)$, fueron 
moderadamente significativas, contribuyendo las variables sobre el mejoramiento de las políticas del Clima organizacional.

Figura 7. Opinión estudiantil sobre el $R d c$ en una Universidad del norte de Sinaloa.

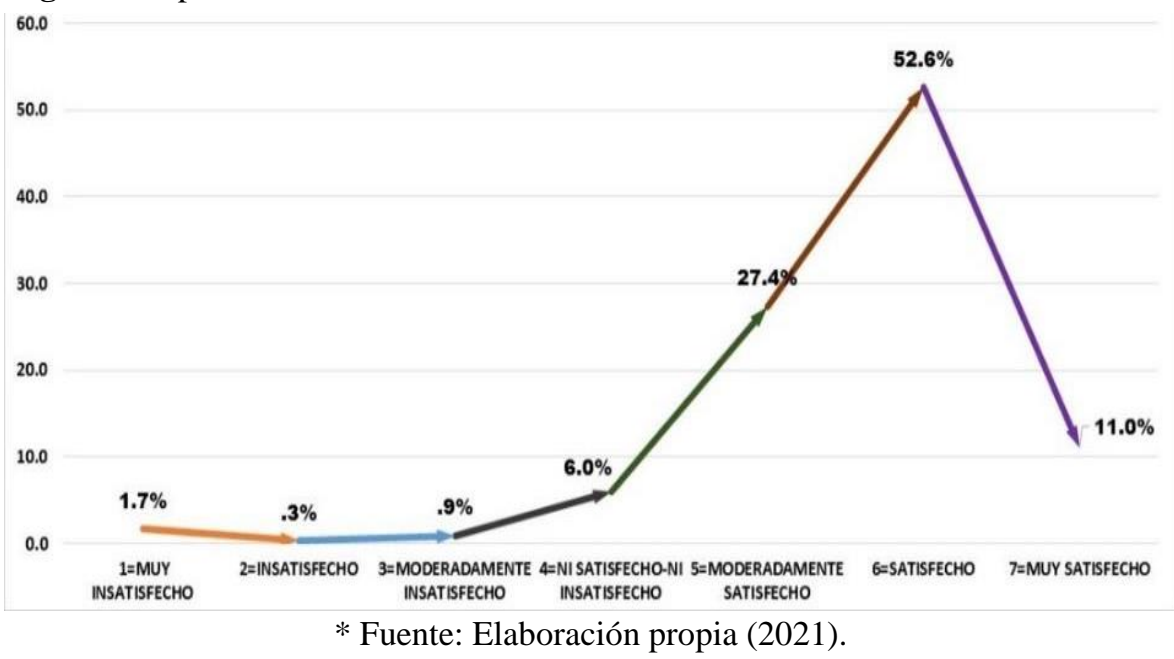

Figura 8. Correlación estadísticamente significativa $(r=0.761 P<0.01)$ entre $R d c$ con $C o$ en estudiantes de una Universidad en un contexto de diversidad cultural.

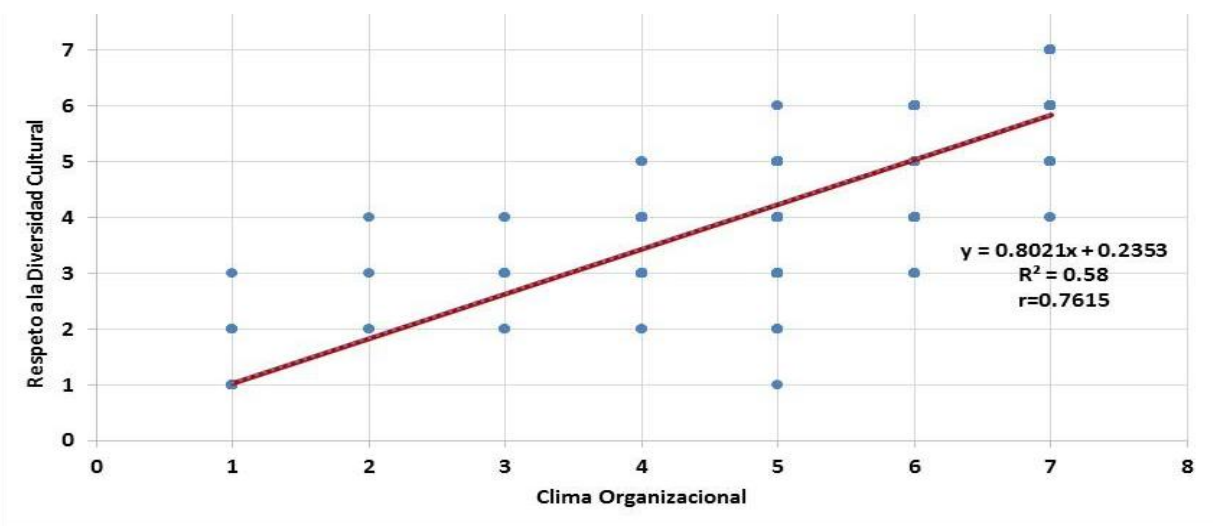

* Fuente: Elaboración propia (2021).

Cohesión (Cn). El 66.5\% está de muy a moderadamente satisfechos ya que perciben que la Universidad promueve la unidad, trabajo en equipo y comunicación entre estudiantes, maestros, autoridades y las demandas son atendidas, lo que en este grupo se percibe que el Co está equilibrado, fortaleciendo la convivencia y avance del Plan de Desarrollo Universitario (Figura 9); 13.1\%, está de muy a insatisfecho ya que no observan planes que fortalezcan la unidad y práctica de valores en un contexto de interculturalidad institucional; $20.3 \%$ son indiferentes, lo que no ayuda a la unidad, la comunicación, la interculturalidad, trabajo en equipo y el deseo de buscar 
ambientes de $C o$ equilibrados que impulsen el desarrollo y la calidad académica.

Correlación entre Cn y Co. La interacción entre $C n$ y $C o$ con el coeficiente de Correlación Lineal de Pearson fue estadísticamente significativa $(r=0.832 \quad P<0.01)$, entendiendo que $C n$ aporta elementos y herramientas al mejoramiento del $C o$ (Figura 10). La variable $C n$ con respecto a $S e(r=0.684 P<0.01), R d c(r=0.627 P<0.01), A f(r=0.628 P<0.01)$ y $E q$ $(r=0.658 P<0.01)$ fueron moderadamente significativos; $C n$ con $G n(r=0.754$ $P<0.01)$ fue estadísticamente significativa, existiendo una dependencia con el Co.

Figura 9. Opinión estudiantil sobre la variable $C n$ en una Universidad dentro de un contexto de diversidad cultural.

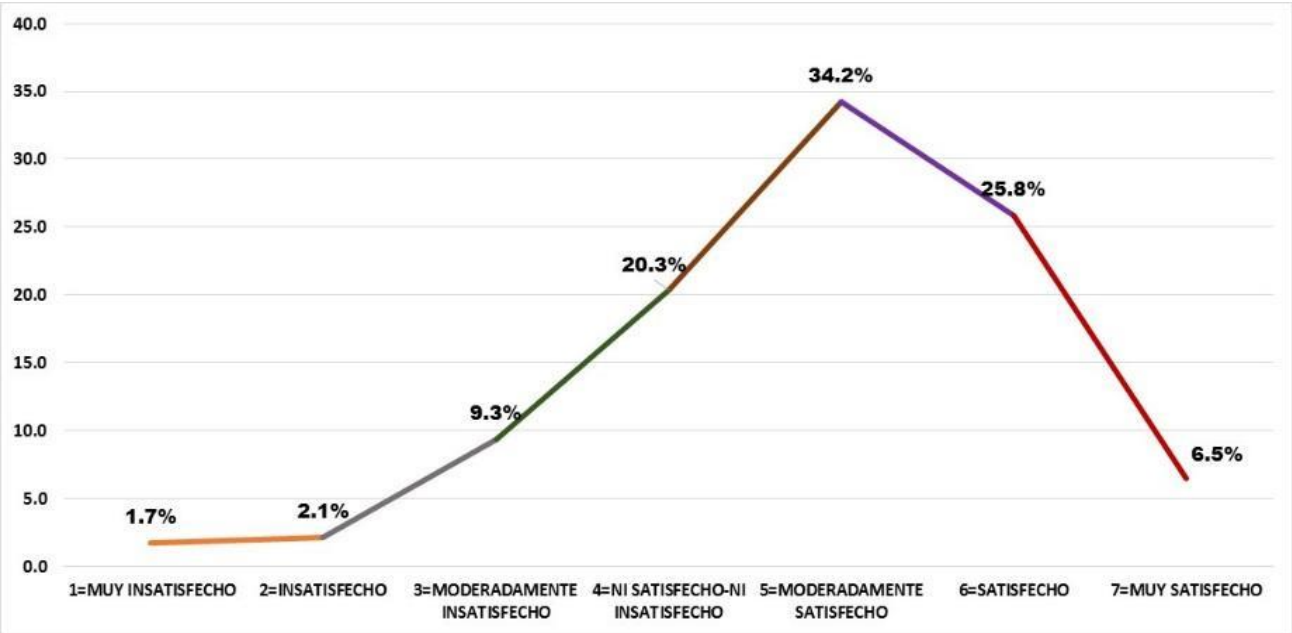

*Fuente: Elaboración propia (2021).

Figura 10. Correlación significativa $(r=0.832 P<0.01)$ entre las variables $C n$ con $C o$ en estudiantes de una Universidad en un contexto de diversidad cultural.

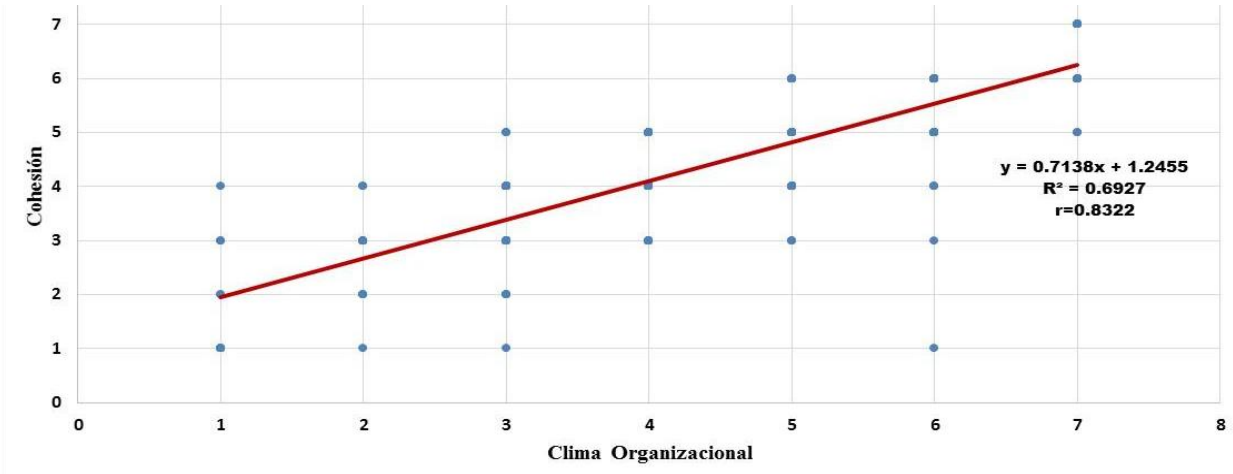

*Fuente: Elaboración propia (2021). 
Gestión (Gn). La respuesta estudiantil de Gn con Co universitario se expone en la (Figura 11). El 62.6\% está de muy a moderadamente satisfechos de cómo se trabaja en la Universidad en el ámbito de la $G n$, siendo importante se mantenga la vinculación hacia fuera del campus y dentro de la Institución con sectores productivos, familiares y el sector público para darle certeza a la vida académica; $21.4 \%$, no le interesa si la universidad está trabajando para vincular actividades hacia otros sectores, consecuencia de la falta de comunicación entre autoridades y la comunidad universitaria sobre planes y proyectos con otros sectores y $16.1 \%$, está de muy a moderadamente insatisfecho de cómo se lleva la gestión. Las autoridades deben esforzarse para informar de proyectos, compromisos, acciones y alternativas para vincular a jóvenes a diferentes sectores (público, privado, comunitario y familiar), lo que asegurará un positivo $C o$ en un ambiente de diversidad cultural.

Figura 11. Opinión estudiantil sobre la variable $G n$ en una Universidad dentro de un contexto de diversidad cultural.

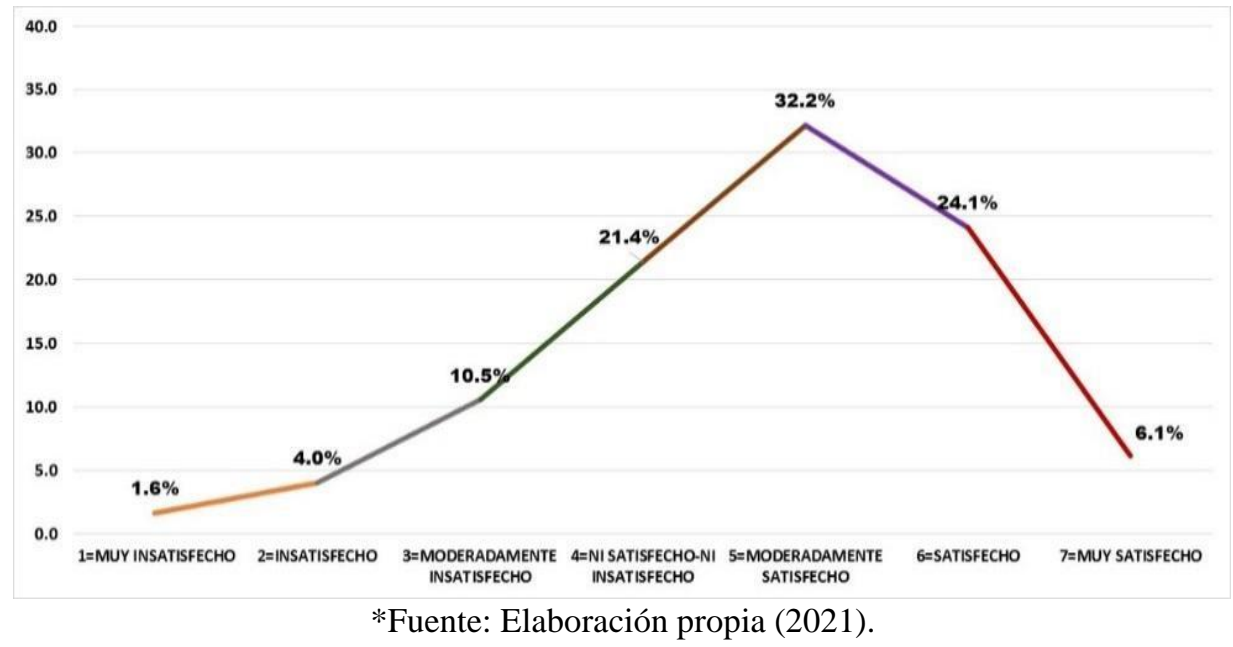

Correlación entre Gn y Co. Fue estadísticamente significativo $(r=0.832 P<0.01)$, aportando la $G n$ elementos y herramientas que fortalecen el Co universitario (Figura 12). 
Figura 12. Correlación estadísticamente significativa $(r=0.832 P<0.01)$ entre variables $G n$ con $C o$ en estudiantes de una Universidad dentro de un contexto de diversidad cultural.

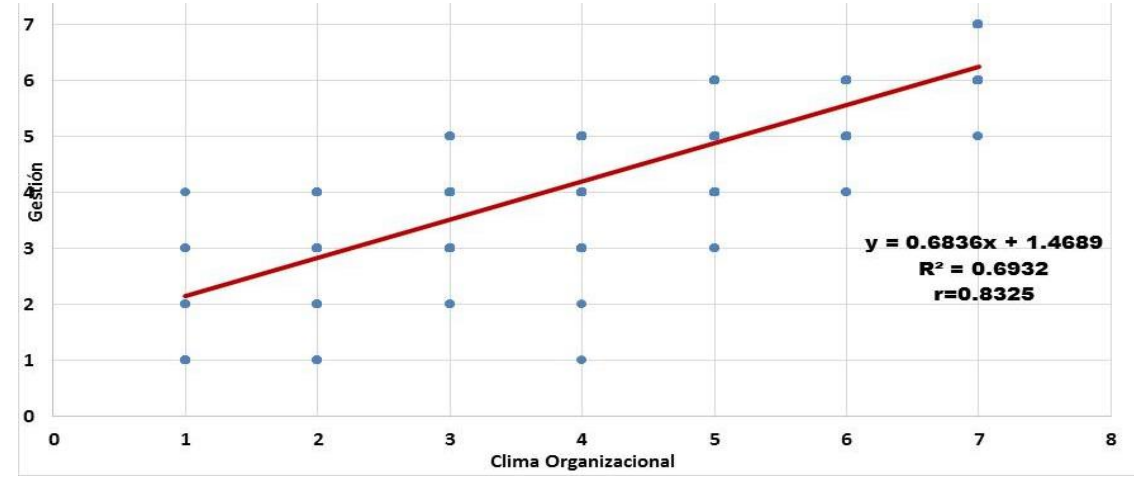

*Fuente: Elaboración propia (2021).

La interacción de la variable $G n$ con $S e(r=0.653 P<0.01), R d c$ $(r=0.564 P<0.01)$, Af $(r=0.666 P<0.01)$ y $E q(r=0.674 P<0.01)$, fueron moderadamente significativos y con $C n(r=0.754 P<0.01)$, estadísticamente significativa.

Área física (Af). El 67.8\%, están de muy a moderadamente satisfechos de cómo se encuentran las instalaciones con mantenimiento y funcionales para grupos vulnerables, suficientemente amplias y confortables para la organización de eventos; $16.4 \%$, no les interesa opinar, argumentando que la autoridad es indiferente en apoyar a grupos vulnerables en cuanto a la adaptación de sus instalaciones; $15.8 \%$ está de muy a moderadamente insatisfecho, señalando que las instalaciones están sucias con falta de mantenimiento, baños en malas condiciones, sin papel y sucios; no existen áreas para asesorías con profesores, llevándose en pasillos, salones de clases, biblioteca y en la cancha deportiva, lo que influye en el deterioro académico y el Co (Figura 13). 
Figura 13. Opinión estudiantil sobre la variable Áf en una Universidad en un contexto de diversidad cultural.

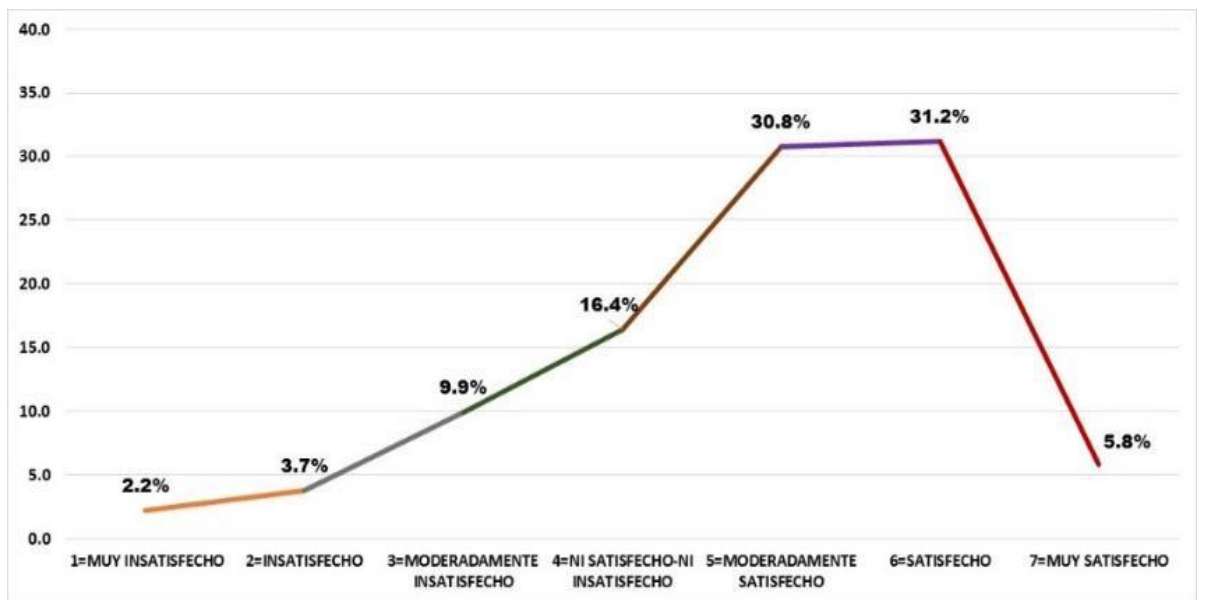

*Fuente: Elaboración propia (2021).

Correlación entre Af $\boldsymbol{y}$ Co. El coeficiente de Correlación fue estadísticamente significativo $(r=0.796 \quad P<0.01)$, aportando la variable $A f$ elementos para el fortalecimiento del $C o$ en la Universidad (Figura 14). Af con Se $(r=0.558 P<0.01), R d c(r=0.563 P<0.01), C n(r=0.628 P<0.01), E q$ $(r=0.698 \quad P<0.01)$ y $G n \quad(r=0.666 \quad P<0.01)$ fueron moderadamente significativos, existiendo respuesta positiva con el Clima organizacional.

Figura 14. Correlación estadísticamente significativa $(r=0.796 P<0.01)$ entre variables $A f$ con $C o$ en estudiantes de una Universidad en un contexto de diversidad cultural.

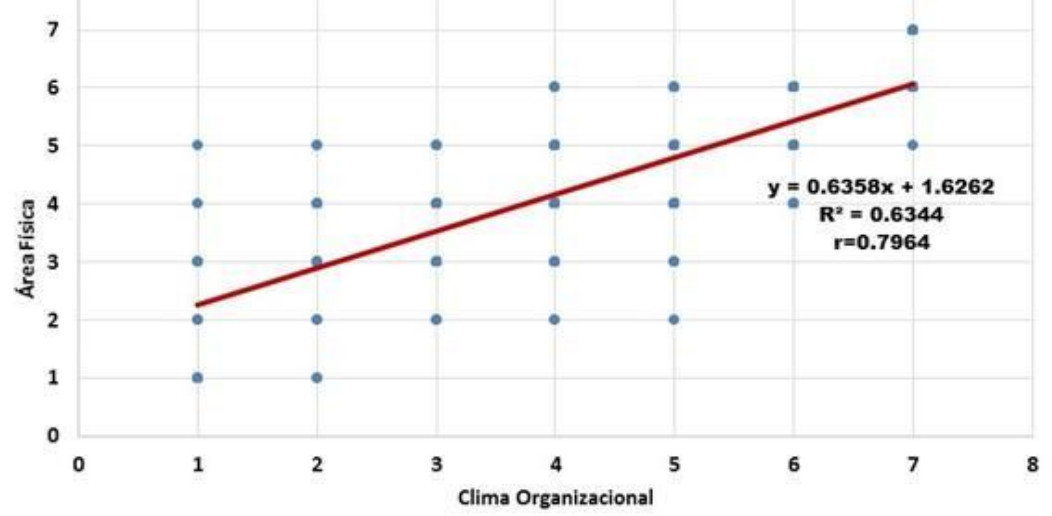

*Fuente: Elaboración propia (2021).

Equidad estudiantil (Eq). El $81.1 \%$ está de muy a moderadamente satisfechos de cómo se percibe la $\boldsymbol{E} \boldsymbol{q}$ y el respeto a los derechos humanos $(D h)$ entre estudiantes, además, ven positivo el apoyo brindado a personas vulnerables ya que la Universidad tiene apertura para jóvenes de zonas urbanas, semi-urbanas, medio rural y de grupos originarios; $10.5 \%$, dijo no 
interesarles estos temas argumentando que la autoridad no está inmersa en estos grupos y no existe respeto a los $D h ; 8.5 \%$, está de muy a moderadamente insatisfechos de cómo se atienden los $D h$ y la falta de voluntad para fomentarlos sin importar condición, origen, religión, forma de pensar, género, entre otros para que sean respetados y tomados en cuenta. Será relevante que se atiendan demandas para construir ambientes propicios que mejoren y fortalezcan el $\mathrm{Co}$ y se busque armonía entre la diversidad existente (Figura 16).

Figura 16. Opinión de estudiantes sobre $E q$ en una Universidad en un contexto de diversidad cultural.

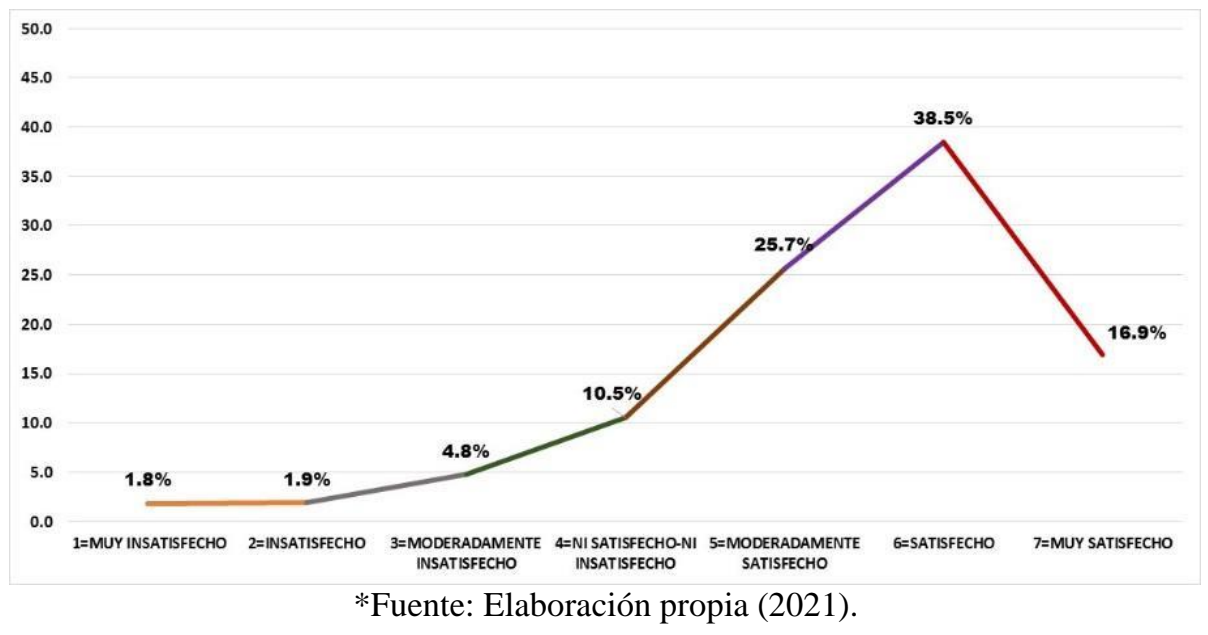

Correlación entre Eq y Co. El coeficiente de Correlación entre estas variables fueron estadísticamente significativos $(r=0.820 \quad P<0.01)$, contribuyendo $E q$ positivamente con el $C o$ (Figura 17). La variable $E q$ con $S e$ $(r=0.625 P<0.01), R d c(r=0.642 P<0.01), C n(r=0.658 P<0.01), G n(r=0.674$ $P<0.01)$ y $A ́ f(r=0.698 \quad P<0.01)$, fueron moderadamente significativos, existiendo correlación directa de estas variables con el Clima organizacional. 
Figura 17. Correlación significativa $(r=0.820 P<0.01)$ entre variables Equidad de género con $C o$ en estudiantes de una Universidad en un contexto de diversidad cultural.

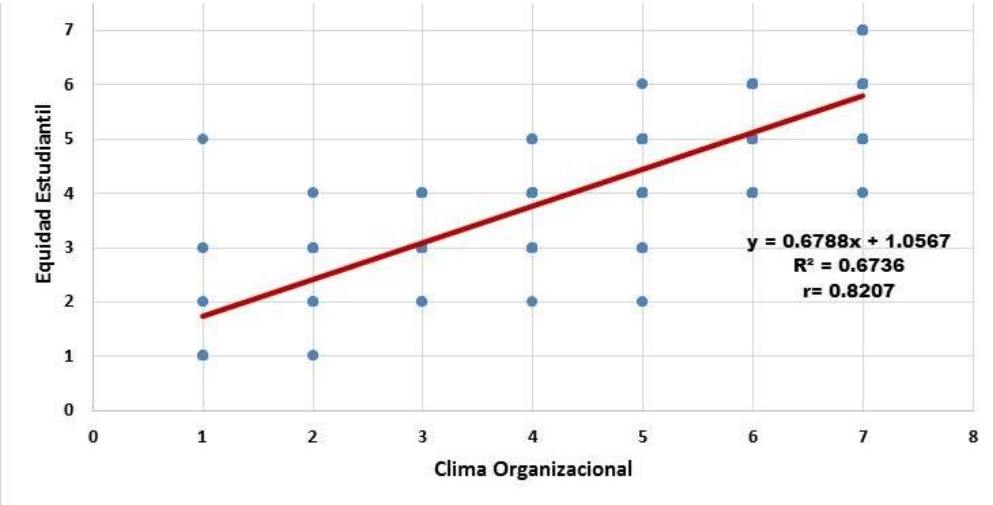

*Fuente: Elaboración propia (2021).

Aceptación o rechazo de hipótesis. De acuerdo a los resultados y a la correlación que mostraron las variables estructurales contempladas con el Clima organizacional $(\mathrm{Co})$, el fomentar estas condiciones en la Universidad entre los estudiantes y la comunidad en general, contribuirá al mejoramiento en la satisfacción, equidad, cohesión, gestión, confort para el desarrollo de las actividades académicas en los jóvenes, por lo que se descarta la Hipótesis nula y se acepta la Hipótesis alternativa según los datos descriptivos y las correlaciones existentes entre las variables independiente y dependiente.

Argumentos finales. Se exponen los resultados a partir del desarrollo de modelos estadísticos quedando como criterio el de $R^{2}$ de las variables como medida de representatividad global. Se determinó que el modelo se apegaba a los datos con los estudiantes donde se muestra una precisión final de $91.8 \%$ (Figura 18).

Resumen de predictores con las variables estructurales y su $R^{2}$. En la (Tabla 6) se presenta el resumen del análisis predictivo fundamentado en la identificación de las relaciones existentes entre variables estructurales con $C o$ en alumnos que sirvió para predecir posibles resultados en futuras situaciones, lo que dependerá del análisis de datos y de la calidad de las suposiciones. 
Figura 18. Resumen del modelo planteado como medida global del estudio de las variables estructurales con el Clima organizacional.

Resumen del modelo

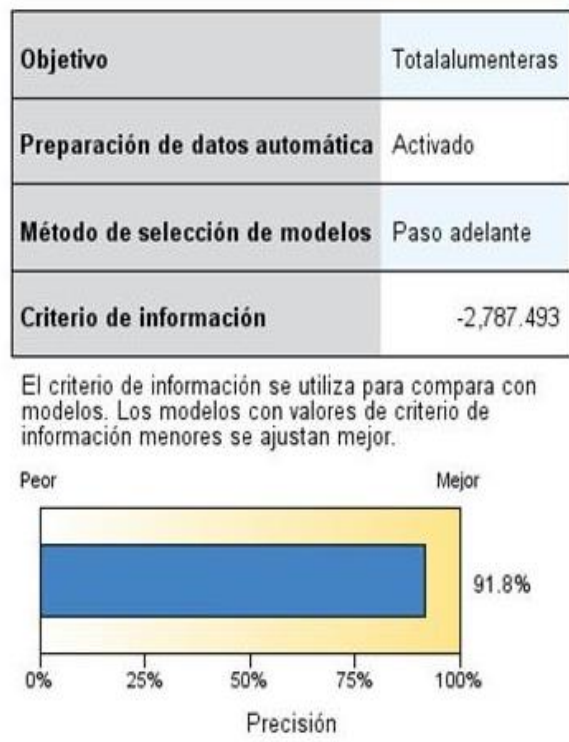

*Fuente: Elaboración propia (2021).

Tabla 6. Resumen del modelo planteado con el resultado de sus predictores.

\begin{tabular}{cccccc}
\hline Modelo & $\mathbf{R}$ & $\begin{array}{c}\mathbf{R} \\
\text { cuadrado }\end{array}$ & $\begin{array}{c}\text { R cuadrado } \\
\text { ajustado }\end{array}$ & $\begin{array}{c}\text { Error estándar } \\
\text { de la estimación }\end{array}$ & Durbin-Watson \\
\hline 1 & $0.832^{\mathrm{a}}$ & 0.693 & 0.693 & 0.58967 & \\
2 & $0.904^{\mathrm{b}}$ & 0.817 & 0.817 & 0.45513 & \\
3 & $0.931^{\mathrm{c}}$ & 0.866 & 0.866 & 0.38943 & \\
4 & $0.944^{\mathrm{d}}$ & 0.891 & 0.891 & 0.35125 & \\
5 & $0.954^{\mathrm{e}}$ & 0.911 & 0.911 & 0.31800 & 0.053 \\
6 & $0.961^{\mathrm{f}}$ & 0.923 & 0.923 & 0.29585 & \\
\hline
\end{tabular}

*Fuente: Elaboración propia (2021).

a. Predictores: (constante), Gestión.

b. Predictores: (constante), Gestión, Equidad Estudiantil.

c. Predictores: (constante), Gestión, Equidad Estudiantil, Respeto a la Diversidad Cultural.

d. Predictores: (constante), Gestión, Equidad Estudiantil, Respeto a la Diversidad Cultural, Área Física.

e. Predictores: (constante), Gestión, Equidad Estudiantil, Respeto a la Diversidad Cultural , Área Física, Servicio a Estudiantes

f. Predictores: (constante), Gestión, Equidad Estudiantil, Respeto a la Diversidad Cultural, Área Física, Servicio a Estudiantes, Cohesión.

$$
Y i^{m}=B o+\beta 1 x_{i}+\beta 2 x_{i}+\beta 3 x_{i}+\beta 4 x_{i}+\beta i 5+\beta 6 x_{i}
$$

Importancia del predictor. De las variables estructurales contempladas en el estudio de $C o$ con estudiantes ( $S e, R d c, C n, G n, A f$ y $E q$ ) 
para la toma de decisiones y conocer cuál es la variable más y la menos importante con el fin de, establecer estrategias de solución en la mejora del Co de la Institución se observan en la (Figura 19). De éstas las más relevantes fueron: Área física; en segundo, Servicio a estudiantes; tercera, Gestión; cuarta, Equidad; quinta, Respeto a la diversidad cultural y la menos relevante Cohesión.

Figura 19. Importancia del predictor que muestran las variables de mayor a menor relevancia de acuerdo a las necesidades de los estudiantes.

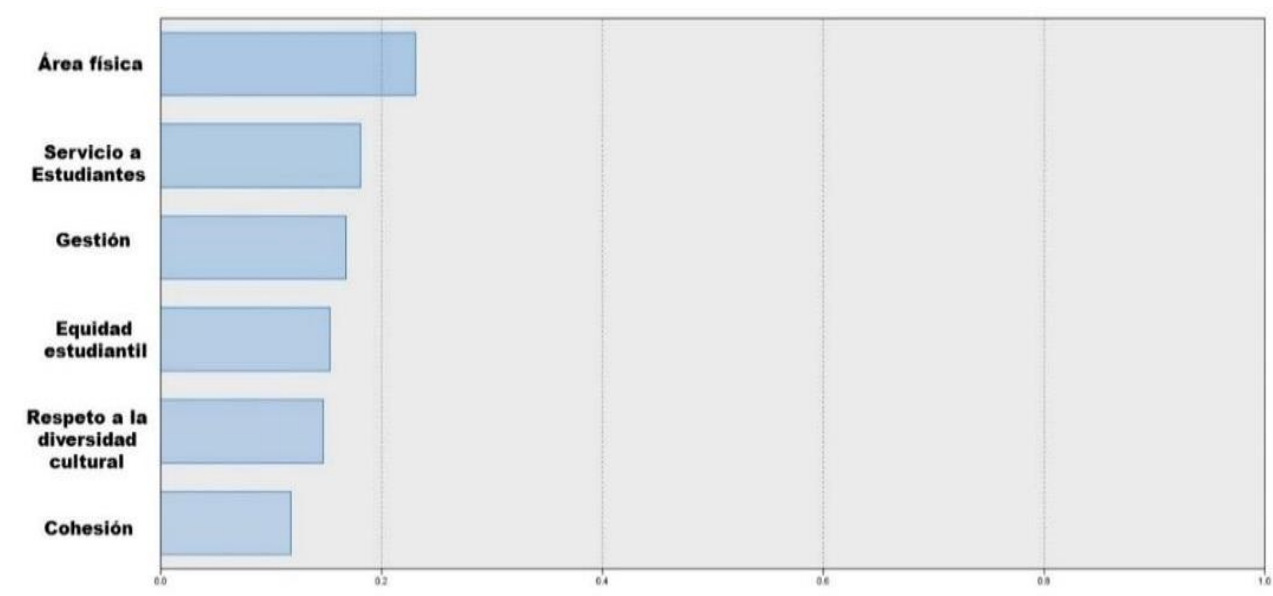

*Fuente: Elaboración propia (2021).

\section{Discusión}

La educación es un derecho de toda persona para mejorar el estatus de vida desde el básico hasta el universitario, así Escamilla et al. (2016) mencionan que quiénes no han logrado alcanzar la educación superior que les permita desarrollarse eficazmente en la vida para trabajar con dignidad y respeto que les permita participar en las actividades cívicas y en general, adquirir nuevos conocimientos basados a sus necesidades, estarán más vulnerables ante fenómenos como la marginación y discriminación. Respecto a la igualdad de género y la búsqueda de derechos políticos, económicos, sociales, educativos, familiares e individuales Fernández-Argüelles et al. (2015) comentan, que existe disparidad debido a la desigualdad entre géneros lo que influye en una marcada inequidad entre hombres y mujeres en un ambiente de diversidad cultural.

La oportunidad que tienen los jóvenes, adultos, grupos originarios y personas de la tercera edad para estudiar en la Universidad CienfuegosMartínez et al. (2016) es una oportunidad para adquirir herramientas permanentes que contrarrestan desventajas económicas, sociales y de preparación entre grupos. Cardona y Zambrano (2014) indica que la interculturalidad debe fomentarse en las instituciones de educación y trabajar con distintos grupos sociales para atender con equidad necesidades 
primordiales. Finalmente en el caso de la lengua, no debe ser problema ya que se debe aprovechar la riqueza que representa la cultura de los grupos originarios y no originarios en el fortalecimiento del Clima organizacional (Co) de cualquier institución educativa (Del Ángel Salazar et al., 2017).

Los estudiantes que combinan varias actividades (trabajo, obligaciones conyugales y otras) con las escolares, según Noriega-Bravo y Pría (2011) investigaron estos fenómenos generados por estudiar y atender múltiples compromisos sobre el desempeño académico, agudizándose con problemas familiares e individuales y en casos extremos, las decisiones por desertar por la incapacidad de enfrentar distintas responsabilidades a la vez. Lo anterior influye importantemente sobre el Co y la identidad del estudiante con su institución, siendo necesario fomentar el espíritu de pertenencia (Posada y Salanova, 2013) que incremente el sentimiento de identidad de grupo en el trabajo académico así como su integración al clima institucional lo que fortalecerá el rendimiento de los jóvenes.

Carrillo-Rivera (2014) indican que la diversidad cultural tiene relevancia en el reconocimiento a la multiculturalidad que motive la convivencia armónica y la comunicación en las universidades, para convertirse en un factor que disminuya la marginación y la exclusión de personas. El respeto a la diversidad cultural será fundamental para promover un agradable $C o$ en las universidades que ayude a la integración del desarrollo humano. Por su parte Sánchez-Tapia (2015) comenta que los valores rigen el comportamiento de las personas incluyendo la "cultura y satisfacción organizacional", términos que involucran valores, necesidades, expectativas, creencias políticas, normas aceptadas y practicadas por los estudiantes; fomento y respeto a la forma de pensar, sentir y creer pues esto estimulará la mayor integración en el $\mathrm{Co}$ dentro de la comunidad estudiantil (Contreras et al., 2011).

Oyarzún y Méndez (2015) resalta la importancia que tiene la Cohesión, porque influye en el fortalecimiento espiritual de grupo y en el desarrollo académico entre equipos cohesivos, estimulando la comunicación, lealtad, amistad en la participación activa en la toma de decisiones comunes y ayuda a generar efectos positivos y satisfactores. Sin embargo Sánchez y Caballero (2011) indican que la $C n$ crea ambientes propicios para la interacción entre grupos y el fomento en el mejoramiento del $C o$ en los centros de estudio.

La Gestión es una parte clave en la promoción de satisfactores para el Co, Segredo et al. (2015) mencionan que el Co representa una herramienta importante en la toma de decisiones, ayudando a proyectar alternativas productivas y de calidad entre integrantes de grupos de trabajos; el mejoramiento continuo y supervivencia de la organización comprendiendo a personas y procesos de desarrollo donde el liderazgo, la reciprocidad, la participación, vinculación y comunicación, son la base para estructurar 
procesos de gestión universitaria con sus integrantes y con sectores externos, públicos o privados, donde vinculen sus acciones para el fortalecimiento de programas y planes de estudio (Sandoval y Pernalete 2014).

Las autoridades universitarias deben hacer esfuerzos y comprometerse para satisfacer necesidades apremiantes de mantenimiento y mejoramiento del Área física, contribuyendo al mejoramiento del Co. El respeto a los derechos humanos para grupos vulnerables debe ser parte de la agenda de actividades para mejorar y adaptar instalaciones para su libre tránsito. Pérez et al. (2013) opinan que la adaptación de la infraestructura incrementará las capacidades y habilidades entre estudiantes. Sin embargo, la inversión aparece como gasto, pero debe ser ante todo aplicado para mejoras, revisando la normatividad universitaria.

La defensa de los derechos humanos según Torres y Vélez (2020) en un ambiente universitario de Equidad estudiantil, forma parte esencial del espíritu intercultural que se debe practicar. Fomentar la igualdad de género, la equidad para garantizar el acceso, permanencia y trato digno en las aulas; el respeto a la diversidad cultural; la búsqueda del diálogo en la solución de conflictos, son garantía para fomentar oportunidades de acceso y permanencia de los jóvenes en la escuela. La diversidad cultural involucra a grupos sociales imaginarios y colectivos, donde se reconoce el carácter pluricultural, multiétnico y hasta multinacional. Velasco (2016) comenta que el respeto a los $D h$ en la diversidad cultural, debe fortalecerse a partir de las necesidades históricas que tiene cualquier institución para vincular a personas, pueblos, comunidades, naciones y civilizaciones en razón del entendimiento y valoración de la multiculturalidad, que se convierta en garantía en el fomento del desarrollo y mejoramiento de su Clima organizacional.

\section{Conclusiones}

El Clima organizacional como pilar fundamental para el fomento de la convivencia entre personas, basados en los resultados obtenidos en la presente investigación, ofrece un panorama amplio de lo que se tiene que hacer para tomar en cuenta a todos los grupos involucrados en la Universidad investigada. Las fortalezas y debilidades entre la comunidad, deben ser motivo de revisiones constantes que ayuden a tomar en cuenta opiniones entre jóvenes, pues son la esencia de la vida activa de la institución. Sería lamentable que solo se tomara en cuenta a las mayorías y se marginara a las minorías en sus peticiones, molestias e indiferencias, con ello, las autoridades universitarias deben hacer esfuerzos para mantener equilibrios entre sectores tomando en cuenta todas las opiniones que fortalezcan el Co que superen conflictos que generalmente se presentan en cualquier universidad. 


\section{References:}

1. Alemán, L. S. y Torres, J. B. (2020). La responsabilidad social de la empresa estatal en el ejercicio pleno de los derechos de los trabajadores en la empresa estatal en Cuba. Rev. IUS. 14 (45): 55-79 pp.

2. Anderson, D. R., D. J. Sweeney, T. A. Williams, J. D. Camm, J. J. Cochran, M. J. Fry and J. W. Ohlmann (2019). Métodos cuantitativos para los negocios. CENAGE. Thirteenth edition.

3. Bolanos, M. J. I., Tumina, J. F. y Ullune, A. C, (2020). Hacia una didáctica artística de colonial. Una propuesta de aula intercultural desde el pueblo misak. Rev. Hist. E Educ. Latinoam. 22 (34): 135-159 pp.

4. Boone, H. N. and Boone, D. A. (2012) Analyzing Likert Data. The Journal of Extension. 50, 1-5 pp.

5. Cabrera, R. I. y T. L. Gallardo (2013). Educación intercultural del estudiante universitario: el enfoque de formación humanístico intercultural. Actualidades Investigativas en Educación. 13 (3): 1-34 pp.

6. Cardona, E. D. y Zambrano R. (2014). Revisión de instrumentos de evaluación de clima organizacional. Estudios Gerenciales. 30: 184$189 \mathrm{pp}$.

7. Castillo, G. E. y Guido G. S. P. (2015). La interculturalidad: ¿principio o fin de la utopía? Revista Colombiana de Educación. 69:17-44 pp.

8. Cienfuegos-Martínez, Y. I., Saldívar-Garduno, A. R. Díaz-Loving y A. D. Avalos-Montoya (2016). Individualismo y colectivismo: caracterización y diferencias entre dos localidades mexicanas. Acta de Investigación Psicológica. 6: 2534-2543 pp.

9. Contreras, F. Barbosa. Juárez, D.F. y Uribe, F. A. (2011). Efectos del liderazgo y del clima organizacional sobre el riesgo psicosocial, como criterio de responsabilidad social, en empresas colombianas del sector salud. Revista Argentina de Clínica Psicológica. 20(1): 173-182 pp.

10. Corral, Y. (2009). Validez y confiabilidad de los instrumentos de investigación para la recolección de datos. Revista Ciencias de la Educación. 19(33): 228-247 pp.

11. Del Ángel Salazar, E. M., Gámez Velázquez, S., Martínez Díaz, N., Meléndez Chávez, S. y Zepeda- Hernández, D. (2017). Clima organizacional del personal docente y administrativo de una institución de educación superior en México. Revista de la Alta Tecnología y Sociedad. 9(2): 79-83 pp.

12. Carrillo-Rivera, A. (2014). Primera Universidad Autónoma Indígena de México y de América Latina. Academia, Ciencia y Cultura: 244$247 \mathrm{pp}$. 
13. Escamilla, F. P. Núñez, P. J. M. y Gómez, T. A. M. (2016). Clima organizacional y sector de pertenencia: un análisis de la percepción de los empleados de entidades deportivas. Revista de Psicología del Deporte. 25: 73-76 pp.

14. Fernández-Argüelles, R., Cobos Díaz, P. A. y Figueroa Varela, M. R. (2015). Evaluación del clima organizacional en un centro de rehabilitación y educación especial. Revista Cubana de Salud Pública. 41(4): 593-602 pp.

15. Figueroa-Céspedes, I. y Yánez-Urbina, C. (2020). Voces y culturas estudiantiles en la escuela: una reflexión teórico-metodológica desde procesos de autoexploración de barreras a la inclusión educativa. Rev. Latinoam. Educ. 14: 109-126 pp.

16. González, M. L., Ortiz, S. J. T. y Gamarra R. L. D. (2020). Dinámicas escolares asociadas a la construcción de un currículo intercultural para la paz y la justicia territorial en el caribe colombiano, 2018-2019. Rev. Hist. Educ. Latinoam. 22 (34): 113-133 pp.

17. González. F. (2017). Por qué medir el clima organizacional. Revista de Negocios. 20(1): 70-71 pp.

18. Noriega-Bravo. V. M. y Pría, M. C. (2011). Instrumento para evaluar el clima organizacional en los grupos de control de vectores. Revista Cubana de Salud Pública, 37(2): 116-122 pp.

19. Núñez, Y. I. y Córdoba, C. A. V. (2020). La educación intercultural bilingüe y sus desafíos para población guaraní de Salta y Misiones (Argentina). RMIE, 25 (85): 419-447 pp.

20. Oyarzún, O. A. y Méndez, M. P. (2015). Proyectos de evaluación de competencias laborales. Pharos. 8(2): 55-120 pp.

21. Pérez, Z. D., M. J. Peralta y D. P. Fernández (2013). Influencia de variables organizacionales en la calidad de vida laboral de funcionarios del sector público de salud en el extremo norte de Chile. Univ. Psychol. 13(2): 541-551 pp.

22. Posada, S. J. A. y S. M. Salanova (2013). Satisfacción laboral: el camino entre el crecimiento psicológico y el desempeño laboral en empresas colombianas industriales y de servicios. Cincel. 13(1): 1-23 pp.

23. Sánchez, F., Suárez, T. y Caballero, A. (2011). Mentiras en el entorno laboral: efectos sobre la confianza y el clima relacional y afectivo. Revista de Psicología del Trabajo y de las Organizaciones. 27(3): 191203 pp.

24. Sánchez -Tapia, M. (2015). La prudencia como sabiduría práctica bajo la perspectiva de Paul Ricœur. La Colmena. (85): 55-67 pp.

25. Sánchez, R. S., Hernández, H. C. A. y Jiménez, G. M. (2016). Análisis de la percepción sobre iniciativa empresarial y el espíritu emprendedor 
en estudiantes de un tecnológico federal. Acta Universitaria. 26(6): 70-82 pp.

26. Sandoval, S.F. y C. D. Pernalete (2014). Marco de trabajo para gestionar las competencias laborales. Revista Venezolana de Información, Tecnología y Conocimiento. 11(3): 11-32 pp.

27. Sagredo, P. A. M., P. J. Pérez, y P. P. López (2015). Construcción y validación de un instrumento para evaluar el clima organizacional en el ámbito de la salud pública. Revista Cubana de Salud Pública, 41(4): 603-619.

28. Torres, G., F. y Vélez, V. G. (2020). Resignificar al otro: configuraciones de la interculturalidad en la formación e intervención del trabajo social. Rev. Hist. Educ. Latinoam. 22 (34):161-180 pp.

29. Velasco, C. S. (2016). Racismo y educación en México. Revista Mexicana de Ciencias Políticas y Sociales. 61(226): 379-407 pp. 